\title{
Hysteresis Process of Gas Hydrate Formation and Dissociation in Pressurized Solution with Dissolved Gases
}

\author{
T. Komai, Y. Yamamoto and K. Ohga* \\ National Institute for Resources and Environment, Tsukuba, Ibaraki, 305 Japan \\ *Faculty of Engineering, Hokkaido University, Kita-ku, Sapporo, O60 Japan
}

\begin{abstract}
The process of gas hydrate formation and dissociation in a pressurized solution has been studied, using a specially designed experimental apparatus. It was found that the phase behavior showed a typical hysteresis due to the kinetic process of hydrate formation and dissociation, and that the environmental conditions for hydrate nucleation were mainly affected by the states of liquid phase. The mechanism of nucleation and crystal growth. of gas hydrate is also discussed based on the change in the clustering structure of the liquid phase.

[Gas hydrate, Clathrate hydrate, Hysteresis process, Crystal growth]
\end{abstract}

\section{Introduction}

Gas Hydrate, a clathrate structure bonded with gas and water molecules, is stable as the solid phase in pressurized solutions with dissolved gases. Phase transitions and equilibria of the gas/water system and gas hydrate crystal have been studied on the basis of the theory of kinetics[1]. However, the dynamic process of their formation and dissociation have not been clarified sufficiently for understanding the nucleation and crystal growth of the gas hydrate[2]. In this paper we present the experimental results on the hysteresis process of gas hydrate formation and dissociation, and discuss the mechanism of the nucleation of hydrate crystal in the liquid phase.

\section{Experimental}

Formation and dissociation processes of gas hydrates were observed and measured, using a high-pressure vessel with an observation system. Fig. 1 shows the experimental set-up and the measuring system. The experimental apparatus consists of a pressure cell, a high-pressure pump, and a control system for both pressure and temperature. The cell was also equipped with magnetic mixing equipment, and three glass windows for the observation of gas hydrate formation. Pure water and methane gas were introduced into the vessel by a high-pressure pump system. Sufficient gas transport into solution was achieved by mixing the interface. The temperature was decreased gradually until the formation of gas hydrate. After growing the hydrate in solution, the temperature was increased to observe the dissociation. Temperatures and pressures in gas and liquid phases were measured and analyzed.

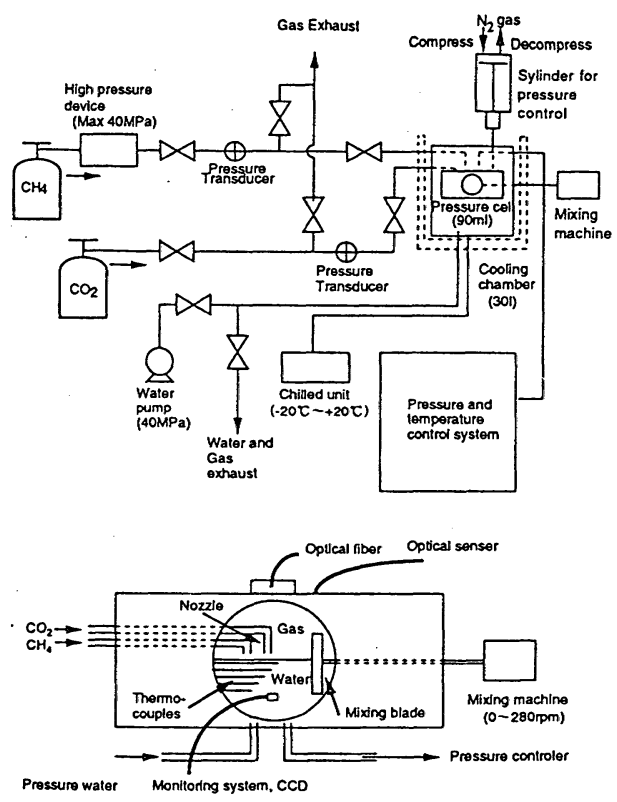

Fig. 1. Experimental apparatus and measuring system.

\section{Results and Discussion}

\subsection{Observation of hydrate formation/dissociation}

The result of an experiment using $100 \%$ methane gas is shown in Fig. 2 as an example of typical trend curves of temperature and pressure. The initial pressure of both gas and liquid phases was $20.0 \mathrm{MPa}$, and the starting temperature was $293 \mathrm{~K}$. The formation of gas hydrate started in the liquid during the period of temperature decrease. With the mixing procedure, numerous fine fragments of methane hydrate appeared around the mixing 
blade in the liquid phase and accumulated in the solution. A rapid increase of temperature due to the heat of formation and a decrease of pressure were observed at the formation point. The pressure gradually decreased due to the changing solubility of the liquid as the temperature dropped. Dissociation of the gas hydrate was observed during the period of temperature increase. After the dissociation point was reached, the temperature declined slightly and then began to rise again. These critical temperatures at which unstable equilibrium conditions arose are important for estimating the phase behavior of gas hydrates[3].

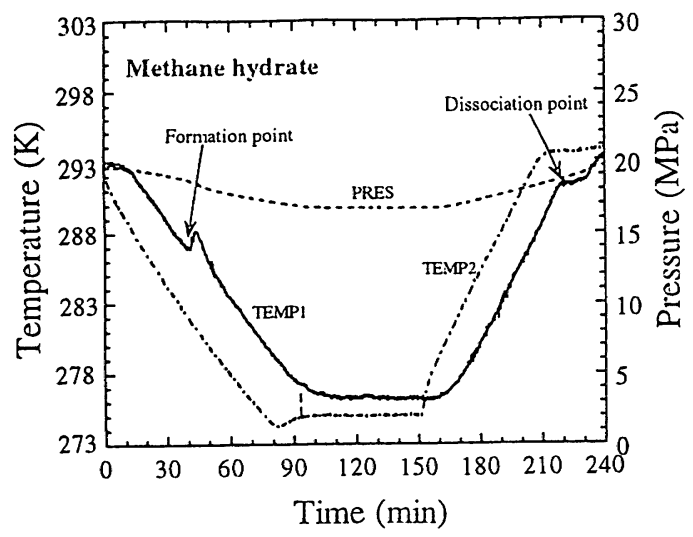

Fig.2. The trend curves of temperature and pressure observed in the experiment of methane hydrate.

\subsection{Phase behavior of gas hydrate}

Fig.3 illustrates the relationship between pressure and temperature in the experiment for methane hydrate. The formation process follows the oscillating curve between a and $\mathrm{d}$, and the dissociation process the curve between $\mathrm{e}$ and f. The figure shows that large differences in temperature and pressure arose in the processes of formation and dissociation of the gas hydrate. The differences of temperature in the formation and dissociation equilibria are usually regarded to be due to the super-cooling effect. In the case of methane hydrate, the temperature difference at supper-cooling and equilibrium states was 2.5 to $4.5 \mathrm{~K}$.

Fig. 4 shows the equilibrium and formation/ dissociation relations obtained for methane hydrate. The upper line represents the relationship between formation temperature $T_{f}$ and pressure $P_{f}$, and the lower line that between dissociation temperature $T_{d}$ and pressure $P_{d}$. The center line corresponds to the phase equilibrium estimated using the thermodynamic theoretical approach. It can be seen that the relation between $\left.P_{f}, P_{d}\right)$ and $\left.T_{f}, T_{d}\right)$ is approximately linear in a semi-log plot. In addition, it was found that the formation relation greatly differs from the theoretical data of phase equilibria. Furthermore, the dissociation relation agrees well with the theoretical equilibrium data. The large differences may reveal interesting phenomena on the mechanism of gas hydrate nucleation in the liquid phase.

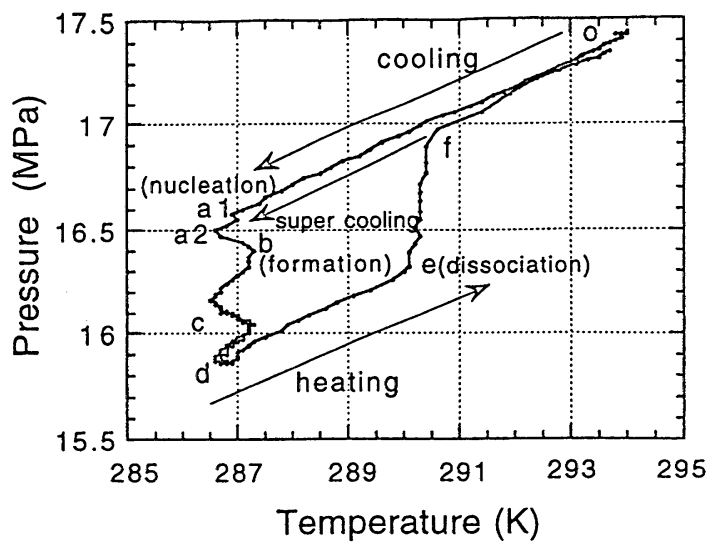

Fig.3. The history curve of pressure and temperature in the process of gas hydrate formation and dissociation.

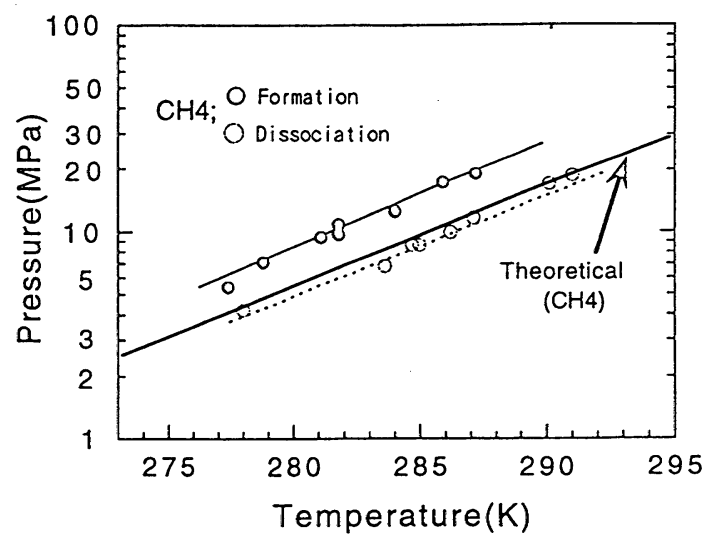

Fig.4 The relations of formation/dissociation pressure and temperature, and the phase equilibrium data.

\subsection{Hysteresis process of gas hydrate}

Fig. 5 shows a typical result of the hysteresis process for methane hydrate formation, in which three continuous experimental runs were conducted under the same liquid and gas conditions. The starting point for each run was 293 $\mathrm{K}$ and $10.0 \mathrm{MPa}$. It is clear that the temperature of the formation point gradually increases with the number of continuous runs. 
Table 1 gives the hysteresis data of $\mathrm{P}$ and $\mathrm{T}_{\mathrm{f}}$ obtained from three continuous runs of the formation and dissociation processes of methane hydrate. It is obvious from these data that the formation temperature tends to increase with the number of repetitions, so that it approaches the equilibrium temperature. This hysteresis process suggests that less activation energy is required to form the hydrate a second time, because of the presence of hydrogen bonds remaining in the solution[4].

Table 1. $\mathrm{P}$ and $\mathrm{T}_{\mathrm{f}}$ data for hysteresis experiment.

\begin{tabular}{|c|c|c|c|c|c|}
\hline & $\begin{array}{l}\mathrm{P} \text { (initial) } \\
(\mathrm{MPa})\end{array}$ & $\begin{array}{l}\mathrm{T}(1 \mathrm{st}) \\
(\mathrm{K})\end{array}$ & $\begin{array}{l}\mathrm{T}(2 \mathrm{nd}) \\
(\mathrm{K})\end{array}$ & $\begin{array}{c}\mathrm{T}(3 \mathrm{rd}) \\
(\mathrm{K})\end{array}$ & $\begin{array}{c}\mathrm{T} \text { (equil) } \\
(\mathrm{K})\end{array}$ \\
\hline & -1 & 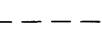 & -1 & - & -- \\
\hline 1. & 10.0 & 277.6 & 280.9 & 281.3 & 283.7 \\
\hline 2. & 15.0 & 283.2 & 285.1 & 285.8 & 287.6 \\
\hline 3. & 20.0 & 287.3 & 288.9 & 290.4 & 291.0 \\
\hline
\end{tabular}

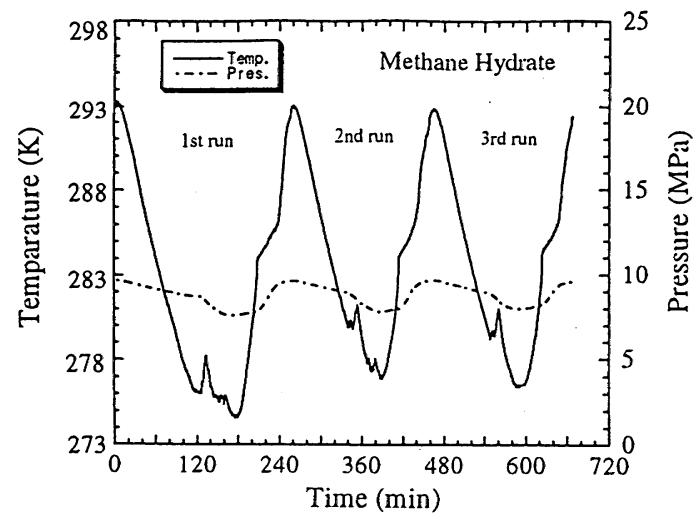

Fig.5. The trend curves of pressure and temperature obtained in the experimental of hysteresis.

\subsection{Mechanism of nucleation of gas hydrates}

Fig. 6 shows the effect of restarting temperature on the formation temperature in the second run for methane hydrate. It is clear that the formation of hydrate is largely affected by the restarting conditions. If the restarting temperature is close to the equilibrium one, most molecular bonds may remain in solution, even above the dissociation temperature. In other words, a significant change in the nucleation process of gas hydrates should be caused by the structure of clusters in the solution. It was reported that the process of nucleation of gas hydrates was affected by the structure of water molecules and heterogeneous conditions in the solution[5]. To clarify this phenomenon, it is necessary to conduct further fundamental research on the structure of water and the relevant changes caused by the presence of guests at the molecular level.

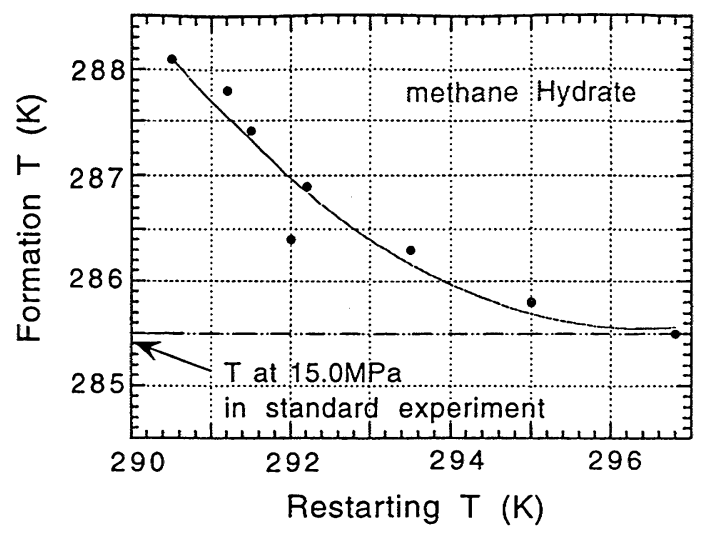

Fig.6. The effect of restarting temperature on the formation temperature.

\section{Conclusions}

We have carried out a series of experiments on the processes of gas hydrate formation and dissociation in pressurized solution. The process was found to exhibit a typical hysteresis behavior due to the super-cooling effect. The environmental conditions for hydrate formation were greatly affected by the states of the liquid phase, the number of runs and the restarting temperature. It is concluded on the basis of the experimental data and discussion, that the effects were caused by the changes in the structure of the aqueous solution at the molecular level.

\section{Acknowledgment}

The authors thank Mr. S. Ikegami and Mr. K. Fukui, graduated students of Hokkaido University, for assistance conducting the experimental work on gas hydrate and the data analysis.

\section{References}

[1] Holder, G.D., Reviews Chem. Eng., 5, 1-70, (1988)

[2] Komai, T. and Yamamoto, Y., 213th ACS Meeting, Fuel Chemistry, 42, 568, (1997)

[3] Schroeter,J.P., Gas Processors Asso., 10, (1982)

[4] Sloan, E.D. and Christiansen, R.L., Int. Conf. Natural Gas Hydrates, 715, 283, (1994)

[5] Yamamoto, Y. and Wakisaka, A., 2nd Int. Conf. Natural Gas Hydrate, 355, (1996) 